\title{
Determination of Arsenic Species in Urine Samples of Subjects Not Exposed to Dietary Arsenic by High Performance Liquid Chromatography- Inductively Coupled Plasma Mass Spectrometry
}

\author{
Jorge Moreda-Piñeiro ${ }^{a}$, Alicia Cantarero-Roldán ${ }^{b}$, Paloma Hermelo-Herbelloc, \\ Antonio Moreda-Piñeiro ${ }^{c}$, and Pilar Bermejo-Barrera ${ }^{c}$ \\ a Grupo Química Analítica Aplicada (QANAP), University Institute of Research in Environmental Studies \\ (IUMA), Centro de Investigaciones Científicas Avanzadas (CICA), Department of Analytical Chemistry. \\ Faculty of Sciences, University of A Coruña, Campus de A Coruña, s/n. 15071 - A Coruña, Spain \\ ${ }^{b}$ Scientific Research Support Services, Edificio de Servizos Centrais de Investigación, \\ University of A Coruña, Campus de A Coruña, s/n 15071 - A Coruña, Spain \\ ${ }^{c}$ Department of Analytical Chemistry, Nutrition and Bromatology, Faculty of Chemistry. University of \\ Santiago de Compostela, Avenida das Ciencias, s/n. 15782 - Santiago de Compostela, Spain
}

\begin{abstract}
Arsenic (As) speciation in human urine was performed by anion exchange chromatography (AEC), followed by inductively coupled plasma mass spectrometry detection (ICP-MS). Separation of arsenite [As(III)], arsenate [As(V)], monomethylarsonic acid [MMA(V)], dimethylarsinic acid [DMA(V], arsenobetaine (AsB), and arsenocholine (AsC) in urine samples was accomplished in 9 minutes, resulting in quantification limits within the $0.3-0.9 \mu \mathrm{g}$ $\mathrm{L}^{-1}$ range. The gradient elution program comprises two different mobile phases consisting of ultrapure water (phase $A$ ) and aqueous $20 \mathrm{mM}$ nitric acid (phase B), both modified with $2 \%$ (v/v) methanol. Sample pre-treatment is based on human urine centrifugation (5000 rpm, $10 \mathrm{~min})$, followed by filtration $(0.45 \mu \mathrm{m})$ and dilution (1:4) with ultrapure water. Relative standard deviations $(\mathrm{N}=11)$ of the overall procedure were $8 \%$ for AsB and DMA(V), 11\% for As(III), and 9\% for MMA(V), As(V), and AsC. Arsenics quantification was per-
\end{abstract}

\section{INTRODUCTION}

Urinary excretion is the major pathway for the elimination of arsenic (As) compounds from the

\footnotetext{
*Corresponding autbor.

E-mail: jorge.moreda@udc.es
}

formed using the standard addition method covering arsenic concentration ranges of $0-100 \mu \mathrm{g} \mathrm{L}^{-1}$ (expressed as arsenic) for all species. Germanium $\left(5 \mu \mathrm{g} \mathrm{L}^{-1}\right)$ was used as an internal standard. Analytical recoveries from the anion exchange column varied from 94 to $101 \%$ (urine sample spiked with low target concentrations), from 99-103\% (urine sample spiked with intermediate target concentrations), and from 97-103\% (urine sample spiked with high target concentrations). The developed procedure has been tested by analyzing the certified reference material NIST CRM 2669 Arsenic Species in Frozen Human Urine which offers certified contents for all arsenic species. After AEC and mass spectrometry detection, MMA(V), DMA(V), AsB, AsC, and also tetramethylarsonium ion (TETRA) were identified in several urine samples. The method has been applied to assess arsenic speciation in several human urine samples collected from volunteers not chronically exposed to arsenic via water and food.

body. Total As and As species concentrations in urine have usually been assessed to study acute or chronic arsenic exposure in humans. The extent of the As metabolism and excretion depends on the chemical forms of the As ingested. Consumption of fish, mollusk, and seaweed can result in a significant intake of arsenic because seafood contains high arsenic levels, generally in the form of organoarsenicals such as arsenobetaine (AsB) and arsenosugars (1-3). After ingestion, several As species such as arsenite [As(III)], arsenate $[\mathrm{As}(\mathrm{V})]$, monomethylarsonic acid [MMA(V)], and dimethylarsinic acid [DMA(V)] are excreted in the urine. Reported data show that AsB, arsenocholine (AsC), trimethylarsine oxide (TMAO), tetramethylarsonium ion (TETRA), and other several unknown species are present in urine after fish and mollusk ingestion (3-5), whereas species such as MMA(V), DMA(V), dimethylarsinoylethanol (DMAE), and other unidentified urinary arsenic species were reported in urine after seaweed ingestion (2,6-8). Recent studies have also shown the presence of the trivalent methylated As forms, monomethylarsonous acid [MMAs(III)] and dimethylarsinous acid [DMAs(III)], in human urine at relatively low concentrations $(9,10)$.

Arsenate ingestion implies a first reduction step, followed by a methylation process of As(III) to MMA(V), and then to DMA(V) $(11,12)$. Methylation does not appear to occur after ingestion of the less toxic As species [MMA(V) or DMA(V)] or the nontoxic As species (AsB and AsC); these forms are excreted unchanged (1). TMAO 
(trimethylamine N-oxide) is a product of the methylation of DMA(III), which is further metabolized to trimethylarsine and subsequently exhaled in the breath as volatile species. Thus, the TMAO concentration in urine is generally lower than the detection limit of most methods (13). The enzymatic and microbial activity in the human body may be responsible for the metabolism of arsenosugars (3). From these data, arsenic speciation in urine is difficult due to the presence of unknown arsenical metabolites and the extremely low concentrations of some As species. In addition, the high salt content in urine and blank contamination can also inhibit the assessment of arsenic species in urine.

Arsenic speciation in environmental and clinical materials is commonly performed by improved separation techniques, mainly high performance liquid chromatography (HPLC), coupled to advanced detection systems such as inductively coupled plasma mass spectrometry (ICP-MS) and flow injection-hydride generation-fluorescence atomic spectrometry (FI-HG-AFS) $(14,15)$. Although FI-HG-AAS is an appealing methodology for As determination (a high sensitivity and inexpensive technique), ICP-MS is also able to quantify As species at nanogramper-milliliter levels, and the decomposition of organo-As species is not required. Therefore, HPLC-ICP-MS, a rapid and simple methodology, can be applied to a large number of samples in routine analysis.

The aim of the current work was the development of a hyphenated anion exchange HPLC-ICP-MS method for assessing As species in human urine. Troubleshooting derived from the high saline matrix of urine samples was also studied and minimized. A blank contamination source was identified and useful guidelines are proposed for the removal of As(V) contamination.
The presence of several As species in urine was also confirmed by Orbitrap mass spectrometry (Thermo Fisher Scientific, USA).

\section{EXPERIMENTAL}

\section{Instrumentation}

HPLC-ICP-MS analysis was performed with a Dionex HPLC UltiMateO 3000 LC (Dionex, Sunnyvale, CA, USA), equipped with a GP50 gradient pump (Dionex), an AS50 thermal compartment (Dionex), and an AS50 autosampler (Dionex). Arsenic separation was carried out with an IonPac AS7 $(250 \mathrm{~mm} x$ $4 \mathrm{~mm}$ i.d.) anion exchange column (Dionex) and an IonPac AG7 guard column (Dionex). The chromatographic system was coupled with a Thermo X Series ICP-MS (Thermo Fisher Scientific Inc., Waltham, MA, USA). The total arsenic content was measured by ICP-MS without using liquid chromatography (LC). An Accela binary pump (Thermo Fisher Scientific), coupled with an Accela autosampler (Thermo Fisher Scientific) and an LTQ-Orbitrap Discovey mass spectrometer (Thermo Fisher Scientific), was used for arsenic species identification. A centrifuge model Hettich Rotina 35R (DJB LabCare, Buckinghamshire, UK) was used for urine separation of the phases.

\section{Reagents and Standard Solutions}

Ultrapure water with a resistance of $18 \mathrm{M} \Omega-\mathrm{cm}$ was obtained from a Milli-Q ${ }^{\circledR}$ purification device (Millipore Corporation, USA). Methanol (gradient grade) was from Merck (Poole, U.K.). Arsenite and arsenate stock standard solutions (1000 $\left.\mathrm{mg} \mathrm{L}^{-1}\right)$ were from Panreac (Barcelona, Spain). Standard solutions of MMA(V), DMA(V), AsB and AsC (1000 $\mathrm{g} \mathrm{L}^{-1}$ ) were prepared by dissolving the appropriate amounts of MMA(V) $\left(\mathrm{CH}_{3} \mathrm{AsO}\left(\mathrm{ONa}_{2}\right) \cdot 6 \mathrm{H}_{2} \mathrm{O}\right)$ (Carlo Erba Milan, Italy); DMA(V) $\left(\mathrm{C}_{2} \mathrm{H}_{6} \mathrm{AsNaO}_{2} \cdot 3 \mathrm{H}_{2} \mathrm{O}\right)$ (Merck); and
AsB $\left(\mathrm{AsC}_{5} \mathrm{H}_{11} \mathrm{O}_{2}\right.$ ) and AsC (AsC $\mathrm{H}_{5} \mathrm{H}_{14} \mathrm{O}$ ), both purchased from Tri Chemical Laboratory, Inc. (Yamanashi, Japan). Standard solutions of MMA(V), DMA(V), AsB and AsC (1000 $\left.\mathrm{g} \mathrm{L}^{-1}\right)$ were prepared by dissolving appropriate amounts of MMA(V) $\left[\left(\mathrm{CH}_{3} \mathrm{AsO}\left(\mathrm{ONa}_{2}\right) \cdot 6 \mathrm{H}_{2} \mathrm{O}\right)\right]$ (Carlo Erba Milan, Italy) and DMA(V) $\left(\mathrm{C}_{2} \mathrm{H}_{6} \mathrm{AsNaO}_{2} \cdot 3 \mathrm{H}_{2} \mathrm{O}\right)$ (Merck) in ultrapure water. Appropriate amounts of $\mathrm{AsB}\left(\mathrm{AsC}_{5} \mathrm{H}_{11} \mathrm{O}_{2}\right)$ and $\mathrm{AsC}\left(\mathrm{AsC}_{5} \mathrm{H}_{14} \mathrm{O}\right)$ (both purchased from Tri Chemical Laboratory Inc., Yamanashi, Japan) were also dissolved in ultrapure water. The standard solutions for organic arsenic were stored in amber glass bottles and kept at $4^{\circ} \mathrm{C}$. Low concentration standards were prepared daily from the stock solutions. Formic acid (98-100\%) was from Merck. NIST CRM 2669 Arsenic Species in Frozen Human Urine was obtained from the National Institute of Standards and Technology (Gaithersburg, MD, USA). Glass amber vials with a white screw cap and bonded PTFE/silicone septa (Waters, Milford, MA, USA) were used. To avoid metal contamination, all glassware and plastic ware was washed and kept in 10\% (v/v) nitric acid for 48 hours, then rinsed several times with ultrapure water before use.

\section{Human Urine Samples}

Human urine samples were collected from anonymous human donors in clean trace-metal-free urine cups. The donors were not chronically exposed to arsenic via water and food or instructed to eat foods containing arsenic (seafood and seaweed). Urine donations were refrigerated at $-20^{\circ} \mathrm{C}$ and protected from light as soon as possible. The urine samples were measured at least 2 months after collection to guarantee the stability of the arsenic species (16).

The human urine samples were centrifuged ( $5000 \mathrm{rpm}$ for $10 \mathrm{~min}$ utes) to remove any residual sedi- 


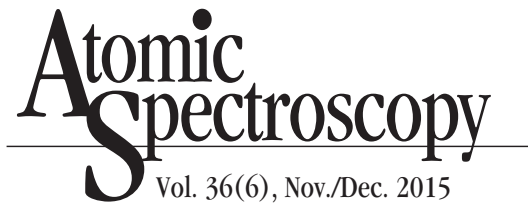

ment. The supernatant was filtered $(0.45 \mu \mathrm{m}$ cellulose acetate syringe filters), diluted (1:4 with ultrapure water), and injected directly into the HPLC-ICP-MS or LC-MS for speciation analysis.

\section{ICP-MS Measurements}

Total arsenic in human urine samples was measured by ICP-MS under the operating conditions listed in Table I. The determinations were performed using the method of standard addition covering arsenic concentrations from 0 to $1000 \mu \mathrm{g} \mathrm{L}^{-1}$. Germanium at a concentration of $5 \mu \mathrm{g} \mathrm{L} \mathrm{L}^{-1}$ was selected as an internal standard. ICP-MS determinations gave a limit of detection (LOD) and a limit of quantification (LOQ) based on the 3 S.D./10 S.D. criterion (S.D. = standard deviation of 11 measurements of a reagent blank) of 0.2 and 0.7 $\mu \mathrm{g} \mathrm{L}^{-1}$, respectively. Accuracy of the method was assessed by analyzing NIST CRM 2669 Arsenic Species in Frozen Human Urine in triplicate. Values of $24.0 \pm 0.8$ and $57.6 \pm 1.1$ $\mu \mathrm{g} \mathrm{L}^{-1}$ (for levels I and II, respectively) were found, which were in good agreement with the certified values of $22.2 \pm 4.8$ and $50.7 \pm 6.3$ $\mu \mathrm{g} \mathrm{L}^{-1}$ (for levels I and II, respectively). This was proven after statistical evaluation by applying a $t$-test (95\% confidence level, seven degrees of freedom), which gave $t_{\text {cal }}$ values of 0.64 and 0.99 (for levels I and II, respectively) lower than the $t_{\mathrm{tab}}$ value of 2.37 . The detection (LOD) and quantification (LOQ) limits, based on the $3 \sigma$ and $10 \sigma$ criterion $(\sigma$, standard deviation of the background signal) expressed as $\mu \mathrm{g} \mathrm{L}^{-1}$, are listed in Table II. Accuracy was assessed by analyzing the NIST CRM 2669 Arsenic Species in Frozen Human Urine (levels I and II). After statistical evaluation by applying the $t$-test at the $95 \%$ confidence level for seven degrees of freedom, good agreement $(\mathrm{N}=8)$ was found between the measured concentrations and the certified values at both levels (Table III).
TABLE I

Anion Exchange HPLC-ICP-MS Conditions

\begin{tabular}{|c|c|c|}
\hline \multirow[t]{4}{*}{ ICP-MS } & Radiofrequency & $1400 \mathrm{~W}$ \\
\hline & Peristaltic pump speed & $2.5 \mathrm{rpm}$ \\
\hline & Nebulizer - MicroMist & $\begin{array}{l}\text { Impact bead (cooled spray } \\
\text { chamber) }\end{array}$ \\
\hline & Nebulizer chamber & $\begin{array}{l}\text { Impact bead, equipped with } \\
\text { Peltier cooling device }\left(5^{\circ} \mathrm{C}\right)\end{array}$ \\
\hline \multirow[t]{3}{*}{ Gas flows } & Plasma & $14.0 \mathrm{~L} \mathrm{~min}^{-1}$ \\
\hline & Auxiliary & $0.8 \mathrm{~L} \mathrm{~min}^{-1}$ \\
\hline & Nebulizer & $0.85 \mathrm{~L} \mathrm{~min}^{-1}$ \\
\hline \multicolumn{3}{|c|}{ Torch alignment } \\
\hline & Horizontal & $117 \mathrm{~mm}$ \\
\hline & Vertical & $317 \mathrm{~mm}$ \\
\hline & Sampling depth & $210 \mathrm{~mm}$ \\
\hline \multirow[t]{8}{*}{ Ion optics } & Extraction & $-102 \mathrm{~V}$ \\
\hline & Lens 1 & $-1150 \mathrm{~V}$ \\
\hline & Lens 2 & $-62 \mathrm{~V}$ \\
\hline & Focus & $-7.8 \mathrm{~V}$ \\
\hline & D1 & $-55.7 \mathrm{~V}$ \\
\hline & $\mathrm{D} 2$ & $-140 \mathrm{~V}$ \\
\hline & Pole bias & $-20 \mathrm{~V}$ \\
\hline & Hexapole bias & $-17 \mathrm{~V}$ \\
\hline CCT & $\mathrm{H}_{2} / \mathrm{He}\left(8 \% \mathrm{H}_{2}\right.$ in $\left.\mathrm{He}\right)$ & $5.85 \mathrm{~mL} \mathrm{\operatorname {min } ^ { - 1 }}$ \\
\hline \multicolumn{3}{|c|}{ Mass-to-charge ratio } \\
\hline & As & 75 \\
\hline & $\begin{array}{l}\text { Ge (internal standard), post- } \\
\text { column addition at } 5 \mu \mathrm{g} \mathrm{L}^{-1}\end{array}$ & 72 \\
\hline \multirow[t]{4}{*}{ HPLC } & \multicolumn{2}{|c|}{$\begin{array}{l}\text { IonPac AS7 ( } 250 \mathrm{~mm} \times 4 \mathrm{~mm} \text { i.d., } 10 \mu \mathrm{m}) \\
\text { anion-exchange column }\end{array}$} \\
\hline & Injection volume & $25 \mu \mathrm{L}$ \\
\hline & Column temperature & $25^{\circ} \mathrm{C}$ \\
\hline & Mobile phase flow rate & $1.35 \mathrm{~mL} \mathrm{~min}^{-1}$ \\
\hline Mobile ph & \multicolumn{2}{|c|}{ Ultrapure water, $1 \%(\mathrm{v} / \mathrm{v})$ methanol } \\
\hline \multicolumn{2}{|c|}{ Mobile phase B } & $80 \mathrm{mM}$ nitric acid ( $\mathrm{pH} 1.3$ ), 1\% (v/v) methanol \\
\hline \multicolumn{3}{|c|}{ Gradient program } \\
\hline & \multicolumn{2}{|c|}{$0 \mathrm{~min}$ to $8.0 \mathrm{~min}, 100 \% \mathrm{~A},(0 \% \mathrm{~B})$} \\
\hline & \multicolumn{2}{|c|}{ Linear gradient to $85 \% \mathrm{~A},(15 \% \mathrm{~B})$} \\
\hline & \multicolumn{2}{|c|}{$8.0 \mathrm{~min}$ to $8.1 \mathrm{~min}, 85 \% \mathrm{~A},(15 \% \mathrm{~B})$} \\
\hline & \multicolumn{2}{|c|}{ Linear gradient to $100 \% \mathrm{~A},(0 \% \mathrm{~B})$} \\
\hline & \multicolumn{2}{|c|}{$8.1 \mathrm{~min}$ to $10 \mathrm{~min}, 100 \% \mathrm{~A},(0 \% \mathrm{~B}$} \\
\hline
\end{tabular}




\section{HPLC-ICP-MS Measurements}

Anion-exchange HPLC was used to obtain the separation of six arsenic species [As(III), As(V), MMA(V), DMA(V), AsB, and AsC] in a single chromatographic run with a gradient elution consisting of ultrapure water and diluted aqueous nitric acid solution as the mobile phases. The anion-exchange HPLC conditions as well as the ICP-MS settings are summarized in Table I. The chromatographic approach of identifying the arsenic species was based on matching the retention times with available standards after spiking of the samples. Different standard addition curves, using germanium $\left(5 \mu \mathrm{g} \mathrm{L}^{-1}\right)$ as an internal standard, were obtained by covering As(III), As(V), MMA(V), DMA(V), AsB, and AsC concentrations of $0,5,10,25,50$, and 100 $\mu \mathrm{g} \mathrm{L}^{-1}$ (expressed as As), respectively. The human urine samples were diluted 4-fold with ultrapure water before injection.

\section{HPLC-Orbitrap MS Measure- ments}

Target arsenic species (MMA(V), DMA(V), AsB, and AsC) present in the urine samples and in NIST CRM 2669 Arsenic Species in Frozen Human Urine were confirmed by HPLC-MS (see conditions listed in Table IV). Detection was performed in the positive ion atmospheric pressure chemical ionization (PI-
APCI) mode. The analyzer was FTMS with a scan range of $\mathrm{m} / \mathrm{z}$ 73.00-180.00 (resolution 30,000). The aqueous standards and the urine samples were matched with mobile phase A [formic acid solution plus $1 \%(\mathrm{v} / \mathrm{v})$ methanol, $\mathrm{pH}$ 2.9].

\section{RESULTS AND DISCUSSION}

\section{Preliminary Studies: Human Urine Sample Dilution, Injection Volume, and As(V) Contamination}

Although anion exchange chromatography coupled to ICP-MS for arsenic speciation and quantification in several matrices works well, there are two important problems when this hyphenated technique is applied to complex samples such as human urine. First, the saline composition of the human urine matrix could produce the co-elution of arsenicals [i.e., co-elution of MMA(V), DMA(V) and AsB or the co-elution of As(V) and AsC] when undiluted urine samples are injected into anion exchange columns. Secondly, the low concentration of some arsenicals [As(III) or MMA(V) or As(V)] in urine requires the analysis of undiluted urine samples or the injection of high injection volumes into HPLC. However, minor arsenical species could be overlapped by major arsenicals (i. e., AsB) when using high injection volumes, and arsenical retention times could also change due to urine sample salinity differences. Finally, large conditioning steps for saline matrix removal from the column are required when injecting undiluted urine samples or when using large injection volumes which increases the total analysis time. These issues can be overcome or minimized by urine dilution with ultrapure water (typically 1:4) using an injection volume

TABLE II

Mean Slopes for Calibration, and LOD and LOQ $(\mathrm{N}=11)$

\begin{tabular}{lccc}
\hline & $\begin{array}{c}\text { Mean Calibration } \\
\text { Slope } \pm \text { S.D. }\end{array}$ & $\begin{array}{c}\text { LOD } \\
\left(\mu \mathrm{g} \mathrm{L}^{-1}\right)\end{array}$ & $\begin{array}{c}\text { LOQ } \\
\left(\mu \mathrm{g} \mathrm{L}^{-1}\right)\end{array}$ \\
\hline Total As & $0.025 \pm 0.01$ & 0.20 & 0.70 \\
As(III) & $4265 \pm 525$ & 0.10 & 0.33 \\
DMA & $6100 \pm 857$ & 0.25 & 0.70 \\
MMA & $6186 \pm 551$ & 0.26 & 0.85 \\
AsB & $5906 \pm 600$ & 0.18 & 0.60 \\
As(V) & $4252 \pm 495$ & 0.16 & 0.53 \\
AsC & $1884 \pm 145$ & 0.13 & 0.44 \\
\hline
\end{tabular}

TABLE III

Results for Total As and As Species Concentrations (expressed as $\mu \mathrm{g} \mathrm{L}^{-1}$ ) in NIST CRM 2669 Arsenic Species in Frozen Human Urine

\begin{tabular}{|c|c|c|c|c|c|c|}
\hline & Certified Value & $\begin{array}{c}\text { Level I } \\
\text { Found Value }\end{array}$ & $\left|t_{\mathrm{cal}}\right|$ & Certified Value & $\begin{array}{c}\text { Level II } \\
\text { Found Value }\end{array}$ & $\left|t_{\mathrm{cal}}\right|$ \\
\hline Total As & $22.2 \pm 4.8$ & $24.0 \pm 0.8$ & 0.64 & $50.7 \pm 6.3$ & $57.6 \pm 1.1$ & 0.99 \\
\hline As(III) & $5.03 \pm 0.31$ & $4.80 \pm 0.40$ & 1.91 & $1.47 \pm 0.1$ & $1.60 \pm 0.20$ & 2.21 \\
\hline DMA & $3.47 \pm 0.41$ & $3.30 \pm 0.30$ & 1.88 & $25.3 \pm 0.7$ & $26.4 \pm 2.3$ & 1.58 \\
\hline MMA & $1.87 \pm 0.39$ & $1.74 \pm 0.20$ & 2.15 & $7.18 \pm 0.5$ & $7.50 \pm 0.50$ & 2.12 \\
\hline AsB & $12.4 \pm 1.9$ & $11.7 \pm 1.10$ & 2.10 & $1.43 \pm 0.08$ & $1.49 \pm 0.10$ & 1.99 \\
\hline $\operatorname{As}(V)$ & $2.41 \pm 0.30$ & $2.27 \pm 0.22$ & 2.11 & $6.16 \pm 0.95$ & $6.40 \pm 0.5$ & 1.59 \\
\hline AsC & $<0.7$ & $-^{\mathrm{a}}$ & & $3.74 \pm 0.35$ & $3.90 \pm 0.30$ & 1.77 \\
\hline
\end{tabular}

${ }^{\mathrm{a}}<$ LOQ 
of $25 \mu \mathrm{L}$ and inserting a conditioning step of 2 minutes after each elution run. By using these conditions, arsenical co-elution and retention time changes are minimized and minor arsenic species can also be quantified.

Blank contamination by $\mathrm{As}(\mathrm{V})$ is a notorious problem in ultra-trace analysis of arsenicals. Furthermore, overestimations and underestimations in the analytical results can occur when blank levels are not stable. Although all glassware and plasticware was washed and kept in $10 \%(\mathrm{v} / \mathrm{v})$ nitric acid for 48 hours, blank contamination by As(V) was observed. The use of clear glass vials was identified as an As(V) contamination source. Thus, the use of amber glass vials are required to eliminate As(V) contamination.

\section{Optimization of As Species Separation by HPLC-ICP-MS}

Anion exchange HPLC conditions were optimized in order to obtain the separation of As(III), As(V), MMA(V), DMA(V), AsB, and AsC in a single chromatographic run. To

TABLE IV

Anion Exchange HPLC-MS Conditions

\begin{tabular}{lll}
\multicolumn{3}{c}{ TABLE IV } \\
& Anion Exchange HPLC-MS Conditions \\
\hline MS & Display voltage & $5 \mathrm{kV}$ \\
& $\mathrm{N}_{2}$ sheath gas / arbitrary units & 50 \\
& Auxiliary gas / arbitrary units & 10 \\
& Capillary voltage & $480 \mathrm{~V}$ \\
& Temperature & $300{ }^{\circ} \mathrm{C}$ \\
& Tube lens offset & $185 \mathrm{~V}$ \\
& Adquisition time & $15 \mathrm{~min}$ \\
HPLC & IonPac AS7 & Anion-exchange column \\
& & $(250 \mathrm{~mm} \mathrm{x} 4 \mathrm{~mm} \mathrm{i.d.)}$ \\
& Injection volume & $10 \mu \mathrm{L}$ \\
& Column temperature & $25{ }^{\circ} \mathrm{C}$ \\
Mobile phase A & Formic acid (pH 2.9), 1\%(v/v) methanol \\
Mobile phase B & Formic acid (pH 1.3), 1\%(v/v) methanol \\
& Gobile phases flow rate & $100 \% \mathrm{~A}, 0 \% \mathrm{~B}, 0 \mathrm{~min}$ \\
& Gradient program & $100 \% \mathrm{~A}, 0 \% \mathrm{~B}, 6 \mathrm{~min}$ \\
& & $10 \% \mathrm{~A}, 90 \% \mathrm{~B}, 10 \mathrm{~min}$ \\
& & $100 \% \mathrm{~A}, 0 \% \mathrm{~B}, 11 \mathrm{~min}$ \\
\hline
\end{tabular}

achieve the best separation for As species in the shortest time, the chromatographic parameters were optimized for the concentration of nitric acid and the percent of methanol in the mobile phases, gradient ramps, flow rate of the mobile phase, and column temperature. Arsenical separation was carried out by using an injection volume of $25 \mu \mathrm{L}$ of urine samples (1:4 diluted with ultrapure water) spiked with $25 \mu \mathrm{g} \mathrm{L}^{-1}$ of As(III), As(V), MMA(V), DMA(V), AsB, and AsC.

As reported, low nitric acid concentrations in the mobile phase are enough to elute some species such as As(III), MMA(V), and DMA(V). However, higher acidity is needed to elute As(V), AsB, and AsC from the ion exchange columns (17). As reported, low nitric acid concentrations $(<20 \mathrm{mM})$ in the mobile phase are enough to elute some species such as As(III), MMA(V), and DMA(V). High nitric acid concentrations $(\sim 80 \mathrm{mM})$ are needed to elute As(V), AsB, and AsC from ion exchange columns (17). However, those nitric concentrations 
concentration of methanol in both mobile phases [from 1 to $4 \%(\mathrm{v} / \mathrm{v})$ ], have no affect on the chromatographic separation. As previously reported, a small proportion of methanol in the mobiles phases improves the chromatographic peaks for arsenicals (17). Therefore, methanol at a concentration of $2 \%$ $(\mathrm{v} / \mathrm{v})$ was used in mobile phases A and B (Table I).

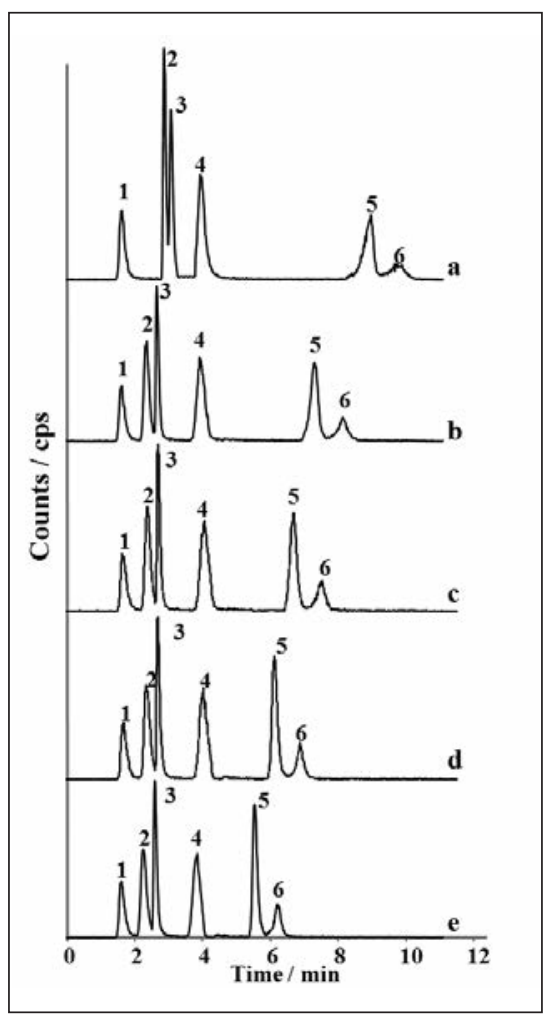

Fig. 1. Chromatograms of arsenic species standards [25 $\mu \mathrm{g} \mathrm{L}^{-1}$ of As(III) (1), DMA(V) (2), MMA(V) (3), AsB (4), As(V) (5), and AsC (6)] added to buman urine (1:4 dilution) for several percentages of mobile phase $B$ : 5\% (a), 10\% (b), 15\% (c), 20\% (d), and $25 \%$ (e). Other HPLC conditions: injection volume of $25 \mu \mathrm{L}$, column temperature of $25^{\circ} \mathrm{C}$, methanol concentration in mobile phase A (ultrapure water) and in mobile phase $B$ of $2.0 \%(v / v)$, phase B composition of $100 \mathrm{mM}$ nitric acid and mobile phase

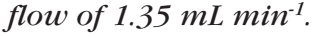

\section{Arsenic Species Confirmation by HPLC-MS}

Orbitrap mass spectrometry was also used to confirm the presence of some organoarsenic species $[\mathrm{MMA}(\mathrm{V}), \mathrm{DMA}(\mathrm{V}), \mathrm{AsB}, \mathrm{AsC}$, and TETRA] in NIST CRM 2669 Arsenic Species in Frozen Human Urine and in some urine samples. Arsenical separation was carried out by using the conditions listed in Table IV and by injecting $10 \mu \mathrm{L}$ of un-spiked NIST CRM 2669 and urine samples (1:4 dilution with ultrapure water).

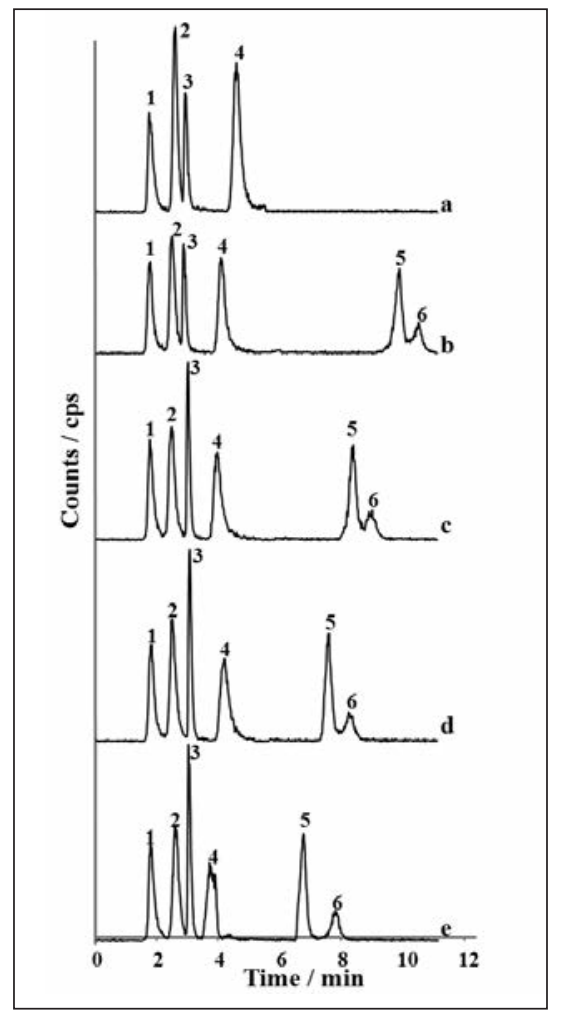

Fig. 2. Chromatograms of arsenic species standards $\left[25 \mu \mathrm{g} \mathrm{L} \mathrm{L}^{-1}\right.$ of As(III) (1), DMA(V) (2), MMA(V) (3), AsB (4), As $(V)$ (5), and AsC (6)] added to buman urine (1:4 dilution) for several nitric acid concentrations in mobile phase B: $20 \mathrm{mM}$ (a), $40 \mathrm{mM}$ (b), $60 \mathrm{mM}$ (c), $80 \mathrm{mM}$ (d) and 100 $m M(e)$. Other HPLC conditions: injection volume of $25 \mu \mathrm{L}$, column temperature of $25^{\circ} \mathrm{C}$, methanol concentration in mobile phase A (ultrapure water) and in mobile phase $B$ of $2.0 \%$ $(v / v)$, percentages of phase $B$ of $15 \%$

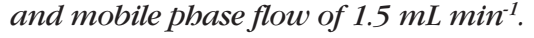

Under these conditions, MMA(V), DMA(V), AsB, and AsC elute at 5.5, $6.5,9.8$, and 12.6 minutes, respectively. The identification was based on $[\mathrm{M}+\mathrm{H}]^{+}$for MMA(V) and DMA(V) at $\mathrm{m} / \mathrm{z}$ ratios of 140.95 and 138.97, respectively; and also on $[\mathrm{M}]^{+}(\mathrm{m} / \mathrm{z}$ ratios of $179.01,165.03$ and 135.06 for AsB, AsC, and TETRA, respectively). Extracted chromatograms at $\mathrm{m} / \mathrm{z}$ ratios of 140.95 [MMA(V)], 138.97 [DMA(V)], 179.01 (AsB), 165.03 (AsC), and 135.06 (TETRA) and the mass

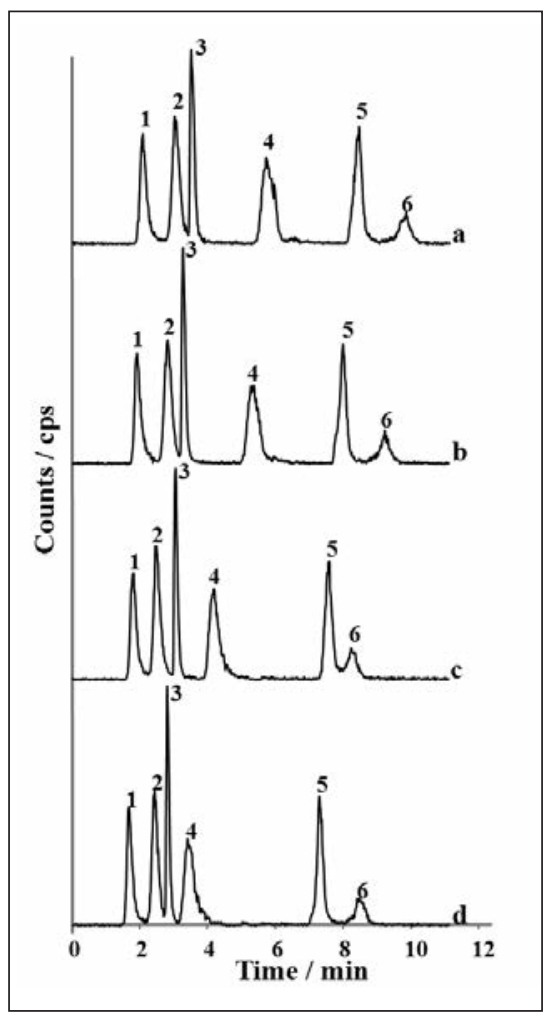

Fig. 3. Chromatograms of arsenic species standards $\left[25 \mu \mathrm{g} \mathrm{L} \mathrm{L}^{-1}\right.$ of As(III) (1), DMA(V) (2), MMA(V) (3), AsB (4), As(V) (5), and AsC (6)] added to buman urine (1:4 dilution) for several mobile phase flows: $1.15 \mathrm{~mL} \mathrm{~min}^{-1}$

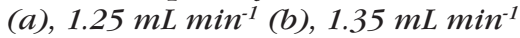
(c), and $1.45 \mathrm{~mL} \mathrm{~min}^{-1}$ (d). Other HPLC conditions: injection volume of $25 \mu \mathrm{L}$, column temperature of $25^{\circ} \mathrm{C}$, methanol concentration in mobile phase A (ultrapure water) and in mobile phase B of $2.0 \%(v / v)$, phase B composition of $80 \mathrm{mM}$ nitric acid and percentages of phase B of $15 \%$. 


\section{A Spectroscopy $_{\text {pemic }}$ \\ $\bigcup$ Vol. 36(6), Nov./Dec. 2015}

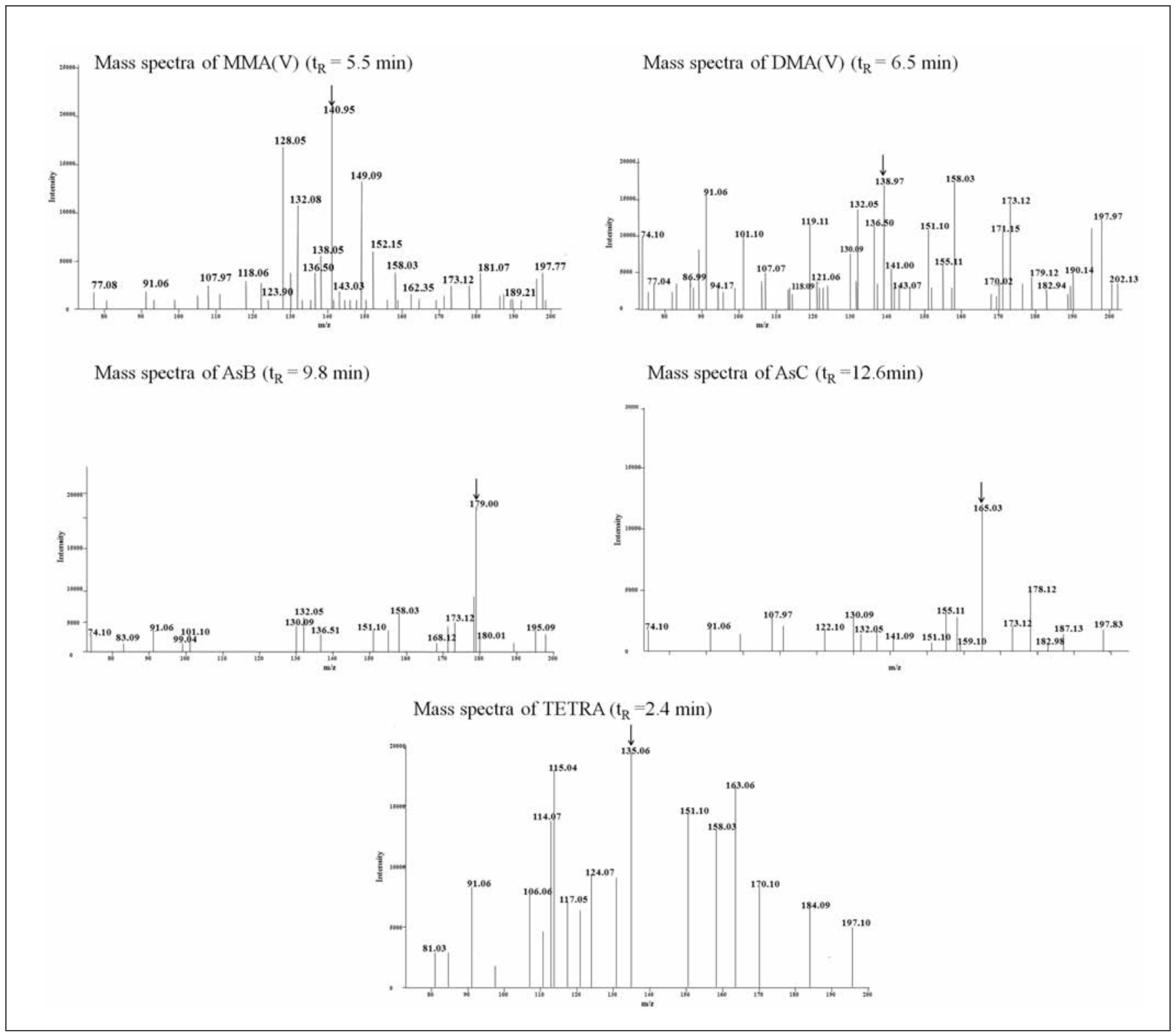

Fig. 4. Mass spectra of MMA(V) ( $m / z$ ratio of 140.95$), D M A(V)(m / z$ ratio of 138.97$)$, AsB $(\mathrm{m} / z$ ratio of 179.01 for AsB), AsC $(\mathrm{m} / \mathrm{z}$ ratio of 165.03$)$, and TETRA $(\mathrm{m} / \mathrm{z}$ ratio of 135.06$)$.

spectra from each chromatographic peak show the presence of MMA(V), DMA(V), AsB, and As(V) in NIST CRM 2669 (levels I and II) and in several human urine samples (Figure 4). This fact is in accordance with the detection/quantification of organoarsenic species in these samples by HPLC-ICP-MS. AsC and TETRA are also confirmed in NIST CRM 2669 (level II) and in several human urine samples (Figure 4).
However, although TMAO is certified in NIST CRM 2669 (level II), a signal regarding this species $(\mathrm{m} / \mathrm{z}=$ 137.05) was not observed.

\section{Analytical Performance}

Different standard addition curves using germanium $\left(5 \mu \mathrm{g} \mathrm{L}^{-1}\right)$ as an internal standard were obtained by covering As(III), As(V), MMA(V), DMA(V), AsB, and AsC concentrations of $0,5,10,25,50$, and $100 \mu \mathrm{g} \mathrm{L}^{-1}$, respectively (expressed as As). Table II lists the means and standard deviations of the slopes of the standard addition graphs for each analyte. The standard addition and internal standard spikes were prepared daily from the stock solutions. Good repeatability of the standard addition curves can be observed over 11 different days, with RSD values around 
$11 \%$ for all cases. In addition, the limit of detection (LOD) and the limit of quantification (LOQ), based on the $3 \sigma$ and $10 \sigma$ criterion $(\sigma$, the standard deviation of the background signal) expressed as $\mu \mathrm{g} \mathrm{L}^{-1}$ (Table II) are low enough to perform As speciation in human urine samples. The LOD and the LOQ were calculated, based on $3 \mathrm{SD} / \mathrm{m}$ and 10 $\mathrm{SD} / \mathrm{m}$, where $\mathrm{SD}$ is the standard deviation of 11 measurements of a blank and $\mathrm{m}$ is the slope of the aqueous calibration graph. Keeping in mind the sample volume, the LODs and LOQs, expressed as $\mu \mathrm{g} \mathrm{L}^{-1}$, are listed in Table II. Accuracy was assessed by analyzing the NIST CRM 2669, which offers two certified concentration levels for As(III), DMA(V), MMA(V), AsB, and As(V). $\mathrm{AC}$ is not certified in level I. Individual arsenic species were identified by comparison of the retention times to standard solutions. Good agreement $(\mathrm{N}=11)$ after statistical evaluation by applying a $t$-test at the $95 \%$ confidence level for 10 degrees of freedom ( $t_{\text {cal }}$ values for all species are lower than the $t_{\mathrm{tab}}$ value of 2.2281) was found between the measured concentrations and the certified contents in both levels (Table III).

Analytical recovery studies were finally performed. Different diluted urine samples were spiked with several concentrations of all arsenic compounds at three levels: $2.5 \mu \mathrm{g} \mathrm{\textrm {L } ^ { - 1 }}$ of As(III), As(V), MMA(V), DMA(V), and $\mathrm{AsC}$, and $25 \mu \mathrm{g} \mathrm{L}^{-1}$ for AsB (low level); $5.0 \mu \mathrm{g} \mathrm{L}^{-1}$ for As(III), As(V), $\operatorname{MMA}(\mathrm{V}), \operatorname{DMA}(\mathrm{V})$, and AsC, and $50 \mu \mathrm{g} \mathrm{L}^{-1}$ for AsB (intermediate level); and $10 \mu \mathrm{g} \mathrm{L}^{-1}$ for As(III), $\operatorname{As}(V), \operatorname{MMA}(V), \operatorname{DMA}(V)$, and AsC, and $100 \mu \mathrm{g} \mathrm{L}^{-1}$ for AsB (high level). Each concentration level was performed seven times; also the unspiked sample was analyzed seven times. High analytical recovery values (94 to $101 \%, 99$ to $103 \%$, and 97 to $103 \%$ for urine samples spiked at low, intermediate, and high levels, respectively) were

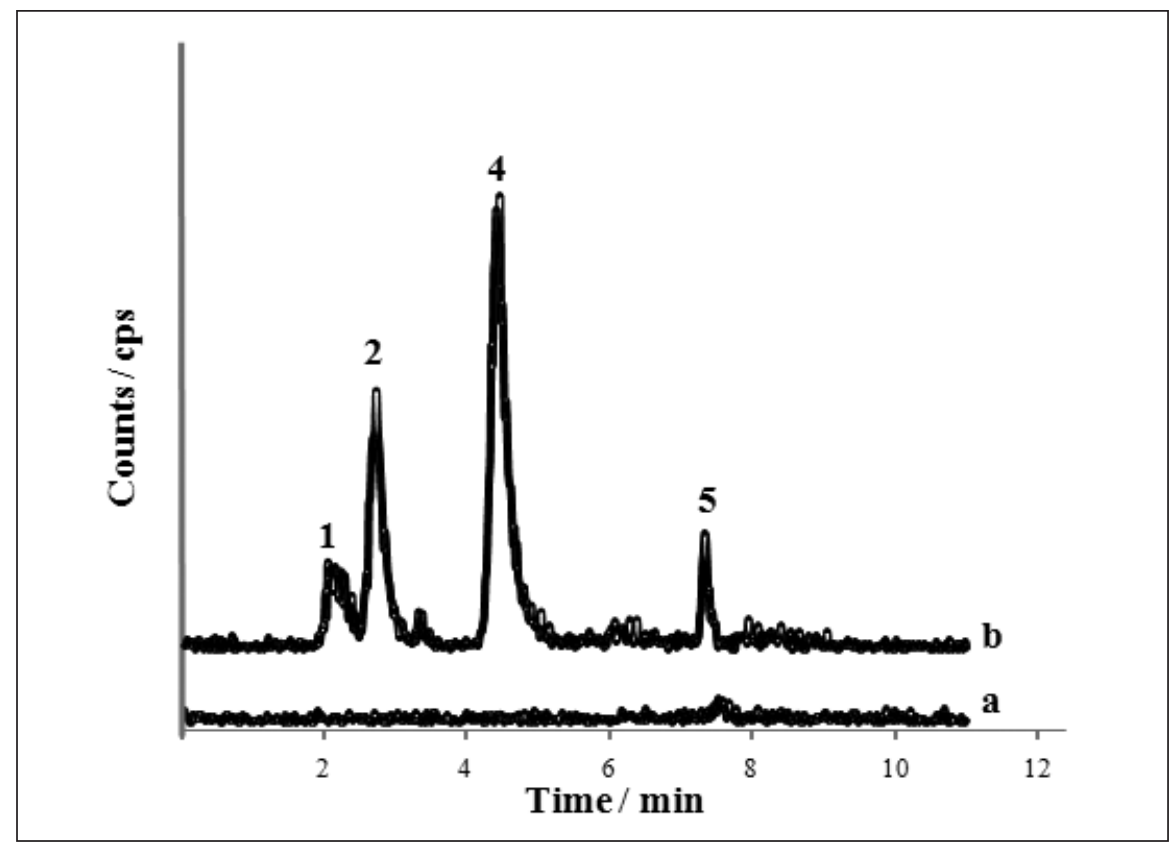

Fig. 5. HPLC-ICP-MS chromatograms of a blank (a) and buman urine (b) (1:4 dilution). As(III) (1), DMA(V) (2), AsB (4) and As(V) (5). HPLC conditions: injection volume of $25 \mu \mathrm{L}$, column temperature of $25^{\circ} \mathrm{C}$, methanol concentration in mobile phase A (ultrapure water), and in mobile phase B of 2.0\% (v/v), phase B composition of $80 \mathrm{mM}$ nitric acid, percentages of phase $B$ of $15 \%$ and mobile phase flow of $1.35 \mathrm{~mL} \mathrm{~min}^{-1}$.

obtained, and good accuracy has been proven.

\section{Applications}

The optimized method was applied to six human urine samples. Each urine sample was measured four times by HPLC-ICP-MS. Typical chromatograms for blank (a) and human urine sample (b) are shown in Figure 5. Table V lists the concentrations of the arsenic species as well as the total arsenic content. It can be seen that AsB was present in all analyzed samples, and AsB was the major arsenic species, which also indicates the consumption of seafood. As(III), MMA(V), DMA(V), and AsC were found in some urine samples, whereas the MMA(V) and As(V) concentrations were lower than the LOQs in most of the analyzed samples. In addition, since the human urine samples were collected from volunteers not instructed to eat seafood, chromatographic signals corresponding to unknown arsenical metabolites were not observed. In addition, since the human urine samples were collected from volunteers also not chronically exposed to arsenic via water and food, chromatographic signals corresponding to unknown arsenical metabolites were not observed. Similarly, trimethylamine N-oxide (TMAO) was not identified in the analyzed urine samples (Figure 5b).

Finally, the arsenical concentrations found in this study were lower than those reported in urine collected from volunteers after ingestion of seafood. It was found that the arsenical concentrations were lower than those reported in urine collected from volunteers after controlled consumption of high amounts of seafood. After ingesting a test meal (blue mussels), total As urinary excretion was $328 \pm 47 \mu \mathrm{g} / \mathrm{L}$, where AsB and DMA 


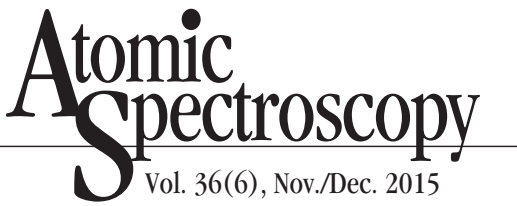

TABLE V

Results in $\mu \mathrm{L} \mathrm{L}^{-1}(\mathrm{~N}=4)$ for As Species and Total As in Different Urine Samples; and $t_{\exp }(95 \%$ confidence level) for Statistical Evaluation of As Species and Total As Concentrations

\begin{tabular}{lcccccc}
\hline & Human Urine 1 & Human Urine 2 & Human Urine 3 & Human Urine 4 & Human Urine 5 & Human Urine 6 \\
\hline As(III) & $1.11 \pm 0.80$ & $1.07 \pm 0.05$ & $<0.33$ & $1.37 \pm 0.07$ & $0.94 \pm 0.04$ & $0.65 \pm 0.03$ \\
DMA & $<0.70$ & $1.19 \pm 0.08$ & $<0.70$ & $<0.70$ & $1.02 \pm 0.08$ & $1.56 \pm 0.11$ \\
MMA & $<0.85$ & $<0.85$ & $<0.85$ & $<0.85$ & $0.91 \pm 0.04$ & $<0.85$ \\
AsB & $20.6 \pm 0.6$ & $43.9 \pm 0.7$ & $76.7 \pm 1.3$ & $68.4 \pm 2.1$ & $33.8 \pm 1.1$ & $14.8 \pm 0.8$ \\
As(V) & $<0.53$ & $<0.53$ & $<0.53$ & $<0.53$ & $<0.53$ & $0.71 \pm 0.02$ \\
AsC & $<0.44$ & $<0.44$ & $0.48 \pm 0.02$ & $0.60 \pm 0.04$ & $0.53 \pm 0.02$ & $<0.44$ \\
Total As & $21.7 \pm 0.61$ & $46.2 \pm 0.71$ & $77.2 \pm 1.3$ & $70.4 \pm 2.1$ & $37.2 \pm 1.1$ & $17.7 \pm 0.81$ \\
Total As $^{\mathrm{b}}$ & $22.2 \pm 1.1$ & $47.2 \pm 2.1$ & $78.3 \pm 2.6$ & $70.6 \pm 3.1$ & $37.6 \pm 1.6$ & $17.8 \pm 1.2$ \\
$\mathrm{t}_{\text {cal }}{ }^{\mathrm{a}}$ & 1.40 & 2.55 & 1.49 & 1.19 & 0.63 & 0.17 \\
\hline
\end{tabular}

${ }^{a}$ Sum of concentrations of As(III), As(V), MMA, DMA, AsB and AsC ( $\left.n=3\right)$. The standard deviation of these sums was obtained by applying the following equation: [SDAs(III), SDDMA, SDMMA, SDAsB, SDAs(V), and SDAsC are the standard deviations $(\mathrm{n}=4)$ ].

b Total As concentration after ICP-MS determination.

${ }^{\mathrm{c}} t_{t \mathrm{ab}}$ (3 degrees of freedom, 95\% confidence range) $=3.18$

accounted for 216.5 and $68.9 \mu \mathrm{g} \mathrm{L} \mathrm{L}^{-1}$, respectively (1). In the same way, after arsenosugar ingestion, the DMA concentrations were found in the 1.5 to $674 \mu \mathrm{g} \mathrm{L}^{-1}$ range in urine (7). It must also be mentioned that the sum of the concentrations for the different arsenic species agrees with the total arsenic concentration found in the samples after ICP-MS quantification. This fact has been confirmed by statistical analysis (Table V).

\section{CONCLUSION}

A fast anionic and cationic species HPLC separation and quantification by ICP-MS has been applied to the arsenicals determination in human urine containing low arsenic levels (urine collected from donors not instructed to eat seafood or seaweed). A chromatographic separation based on a gradient elution by using aqueous solutions containing low nitric acid concentrations as mobile phases has been found effective and useful to elute six arsenicals species in a single chromatographic run. The time required to complete the analysis is approximately 9 minutes, a shorter time than required by other reported chromatographic separations. The described analytical method has fairly good precision and accuracy as well as low quantification limits, which meets the requirements for arsenical species determination in urine samples. Troubleshooting related to the high saline matrix of urine samples was minimized after applying a 1:4 dilution to the human urine samples and by using an injection volume of $25 \mu \mathrm{L}$. The presence of several arsenicals [MMA(V), DMA(V), AsB, AsC, and TETRA] in NIST CRM 2669 Arsenic Species in Frozen Human Urine and the human urine samples was confirmed by HPLC-ICP-MS.

\section{ACKNOWLEDGMENT}

The authors acknowledge the financial assistance of Xunta de Galicia (Programa de Consolidación y Estructuración de Unidades de Investigación Competitivas 2014$2017,2014 / 52$ ) for financial support. We are grateful to Servicios Xerais de Apoio a Investigación at the University of A Coruña for ICPMS technical support.

Received May 29, 2015.

\section{REFERENCES}

1. M. Molin, T.A. Ydersbond, S.M. Ulven, M. Holck, L. Dahl, J.J. Sloth, D. Fliegeld, W. Goessler, J. Alexander and H.M. Meltzer, Food Chem. Toxicol. 50, 2462 (2012).

2. M. Van Hulle, C. Zhang, B. Schotte, L. Mees, F. Vanhaecke, R. Vanholder, X. R. Zhang and R. 3 Cornelis, J. Anal. At. Spectrom. 19, 58 (2004).

3. X.-C. Le, W. R. Cullen, and K. J. Reimer, Clin. Chem. 40, 617 (1994).

4 . V.W.M. Lai, Y.M. Sun, E. Ting, W. R. Cullen, and K. J. Reimer, Toxicol. Appl. Pharmacol. 198, 297 (2004).

5. T. Nakazato, T. Taniguchi, H. Tao, M. Tominaga, and A. Miyazaki, J. Anal. At. Spectrom. 15, 1546 (2000).

6. X.-C. Le, and M. Ma, Anal. Chem. 70, 1926 (1998).

7 . K. A. Francesconi, R.Tanggaard, C. J. McKenzie, and W. Goessler, Clin. Chem. 48, 92 (2002).

8. A. Hata, Y. Endo, Y. Nakajima, M. Ikebe, M. Ogawa, N. Fujitani, and G. Endo, J. Occup. Health 49, 217 (2007).

9. S. Rabieh, A. V. Hirner, and J. Matschullat, J. Anal. At. Spectrom. 23, 544 (2008). 
10. L. Yehiayan, M. Pattabiraman, K. Kavallieratos, X. Wang, L. H. Boise, and Y. Cai, J. Anal. At. Spectrom. 24, 1397 (2009).

11. C. Hopenhayn-Rich, M. L. Biggs, D. A. Kalman, L. E. Moore, and A. H. Smith, Environ. Health Perspect. 104, 1200 (1996).

12 . O. L. Valenzuela, V. H. BorjaAburto, G. G. García-Vargas, M. B. Cruz-González, E. A. García-Montalvo, E. S. Calderón-Aranda, and L. M. Del Razo, Environ. Health Perspect. 113, 250 (2005).

13. X. C. Le, X. Lu, M. Ma, W.R. Cullen, H.V. Aposhian, and B. Zheng, Anal. Chem. 72, 5172 (2000).

14. E. Terlecka, Environ. Monitor. Assess. 107, 259 (2005).

15. M. Leermakers, W. Baeyens, M. De Gieter, B. Smedts, C. Meert, H. C. De Bisschop, R. Morabito, and $\mathrm{Ph}$. Quevauviller, Trends Anal. Chem. 25, 1 (2006).

16. J. Feldmann, V.W.M. Lai, W.R. Cullen, M. Ma, X. Lu, and X.C. Le, Clin. Chem. 45, 1988 (1999).

17. A. Moreda-Piñeiro, E. PeñaVázquez, P. Hermelo-Herbello, P. Bermejo-Barrera, J. Moreda-Piñeiro, E. Alonso-Rodríguez, S. MuniateguiLorenzo, P. López-Mahía, and D. Prada-Rodríguez, Anal. Chem. 80, 9272 (2008). 